\title{
Electrooxidation of Vanillyl Alcohol in Acidic Aqueous Solution Using Rotating Ring-Disk Electrode Voltammetry
}

\author{
Shin-ya KISHIOKA ${ }^{\dagger}$ and Akifumi YaMADA \\ Department of Chemistry, Nagaoka University of Technology, Kamitomioka, Nagaoka, \\ Niigata 940-2188, Japan
}

\begin{abstract}
The electrooxidation of benzylic alcohol derivative in acidic aqueous solution shows an oxidation pre-peak in the cyclic voltammogram, which means that the reaction is proceeding via an ECE mechanism where the second electron transfer occurs at a less positive potential. From the result of the rotating ring-disk electrode voltammetry, the initial oxidation response of the electrode reaction can be extracted.
\end{abstract}

(Received December 8, 2004; Accepted January 28, 2005)

\section{Introduction}

Methoxy-substituted benzyl alcohols are typical representative secondary metabolites of the lignin biodegradation by the whiterot fungus $P$. chrysosporium. ${ }^{1,2}$ In particular, veratryl alcohol (3,4-dimethoxybenzyl alcohol, VA) is a well-known compound that is an effective natural redox mediator. Furthermore, the formation of the corresponding aldehyde, veratryl aldehyde, by a two-electron oxidation has been widely accepted as the activity index of the enzyme. ${ }^{3,4}$ The oxidation mechanism in the above enzymatic reaction has been proposed to be almost the same process as that in the anodic oxidation of VA at electrodes. ${ }^{5,6}$ These reactions can be regarded as ECE (electronchemical-electron) or ECEC mechanisms. However, the cyclic voltammograms of the VA oxidation indicates only one irreversible peak in a conventional time scale, probably because of the short lifetime of intermediates. Therefore, it is difficult to find what value the less positive redox potential VA./VA ${ }^{+}$ couple has. In order to understand the redox mechanism, the information about the redox potential is essential and the value of VA/VA.+ ${ }^{+}$couple has been reported. ${ }^{7,8}$ On the other hand, little information about the redox properties of VA. has been reported, less than that even about other benzyl alcohol derivatives.

In this study, we employed vanillyl alcohol as an alternative model compound for the reaction of methoxy-substituted benzyl alcohols, because the cyclic voltammogram obtained for the vanillyl alcohol oxidation shows a well-defined reduction current response under the conditions of a conventional time scale, as is distinctive for the VA case. ${ }^{9}$ Vanillyl alcohol and the corresponding 2-electron oxidized product, vanillin, have been widely used as natural flavoring reagents. ${ }^{10}$ Though the mediation property of the former has not yet been reported, presumably because its redox potential is low compared to that of VA, the latter is already known as a metabolite during the lignin degradation process. ${ }^{11}$ For the analysis of the reaction mechanism, which involves the short-lived intermediates electrogenerated in solution, rotating ring-disk electrode

$\doteqdot$ To whom correspondence should be addressed.
(RRDE) voltammetry is a powerful tool. In a series of papers, Prater and Bard reported digital simulation treatments of the RRDE in the presence of following chemical reactions (an EC mechanism), an additional electron transfer reaction (an ECE mechanism) and a catalytic reaction, in which the second electron transfer step occurs at the same potential as the first. ${ }^{12,13}$ We treat the reaction of the vanillyl alcohol oxidation as following the ECE and ECEC mechanisms in which the second oxidation step occurs at a less positive potential, and we attempt to detect an intermediate in order to elucidate the electrode reaction of the methoxy-substituted benzyl alcohol in an acidic aqueous medium.

\section{Experimental}

Vanillyl alcohol (4-hydroxy-3-methoxybenzyl alcohol, VNA, Tokyo Kasei Kogyo Co., Ltd., Japan) of reagent grade was used without further purification. Sodium sulfate (reagent grade, Wako Pure Chemical Industries, Ltd., Japan) was used as the supporting electrolyte $\left(0.2 \mathrm{M}\left(\mathrm{mol} \mathrm{dm}^{-3}\right)\right)$ for the electrochemical measurements. The $\mathrm{pH}$ of the electrolytic solutions was adjusted to 1.0 with sulfuric acid (reagent grade, Wako Pure Chemical). All solutions were prepared using highly-purified water from a Direct-Q system (Millipore Co., Ltd.).

The cyclic and the steady-state RRDE voltammetries were carried out using the conventional three-electrode cell configuration. For all the electrochemical experiments, the electrode potential was controlled by a dual potentiostat (HR101B, Hokuto Denko Co., Ltd., Japan) and a function generator (HB-104, Hokuto Denko), and the voltammograms were recorded on an XYY recorder (D72BP, Riken Denshi Co., Ltd., Japan). A platinum disk electrode, having a disk radius of 0.05 $\mathrm{cm}$ and a platinum Teflon rotating ring-disk electrode, having a disk radius $\left(r_{1}\right)$ of $0.364 \mathrm{~cm}$ and inner $\left(r_{2}\right)$ and outer $\left(r_{3}\right)$ ring radii of 0.378 and $0.484 \mathrm{~cm}$, respectively (Hokuto Denko) were used as the working electrodes. The RRDE was rotated using a controller (HR-101A, Hokuto Denko). The collection efficiency, $N$, for the RRDE was determined from the geometry and by experimental measurements of the electrochemical systems involving the reaction of the species electrogenerated at 


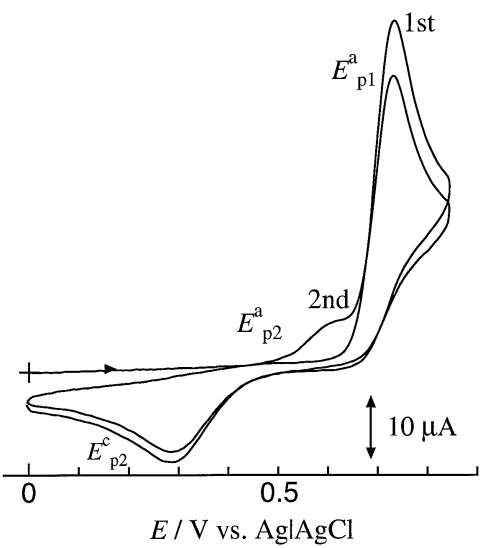

Fig. 1 Cyclic voltammogram for the oxidation of $2 \mathrm{mM}$ vanillyl alcohol in an aqueous solution containing $0.2 \mathrm{M} \mathrm{Na}_{2} \mathrm{SO}_{4}+\mathrm{H}_{2} \mathrm{SO}_{4}$ $(\mathrm{pH} 1.0)$ at a Pt disk electrode at sweep rate of $0.2 \mathrm{~V} \mathrm{~s}^{-1}$.

the disk $\left(\mathrm{K}_{4} \mathrm{Fe}(\mathrm{CN})_{6} / \mathrm{K}_{3} \mathrm{Fe}(\mathrm{CN})_{6}\right.$ couple), the $N$ value was found to be $0.347 \pm 0.001$. A Pt wire was used as the auxiliary electrode and an $\mathrm{Ag}|\mathrm{AgCl}| 3 \mathrm{M} \mathrm{NaCl}$ electrode was the reference electrode. The surfaces of the working disk and RRD electrodes were polished on lapping films (grain sizes of 9, 6, 3, 2, 1 and $0.5 \mu \mathrm{m}$, Sumitomo 3M, Ltd., Japan) and then sonicated in water for $3 \mathrm{~min}$. Argon gas was directly bubbled into the cell solution for $10 \mathrm{~min}$ to remove oxygen from the electrolytic solution, and then flushed over the cell solution throughout the measurements. All experiments were performed at room temperature $\left(25 \pm 1^{\circ} \mathrm{C}\right)$.

\section{Results and Discussion}

Figure 1 shows the typical cyclic voltammograms $(\mathrm{CVs})$ for the oxidation of $2 \mathrm{mM}$ vanillyl alcohol (VNA) in an aqueous solution containing $0.2 \mathrm{M} \mathrm{Na}_{2} \mathrm{SO}_{4}+\mathrm{H}_{2} \mathrm{SO}_{4}(\mathrm{pH} 1.0)$ at a Pt disk electrode. During the initial sweep, the CV of VNA shows a large oxidation peak at $0.75 \mathrm{~V}\left(E_{\mathrm{p}_{1}}^{\mathrm{a}}\right)$. After the potential is reversed at $0.85 \mathrm{~V}\left(E_{\lambda}\right)$, a smaller but well-defined reduction peak is observed at $0.29 \mathrm{~V}\left(E_{\mathrm{p} 2}^{\mathrm{c}}\right)$. When the potential sweep is continuously repeated between $0 \mathrm{~V}$ and $0.85 \mathrm{~V}$, the oxidation pre-peak at $0.6 \mathrm{~V}\left(E_{\mathrm{p}_{2}}^{\mathrm{a}}\right)$ appears and the main peak current of the subsequent sweep at $0.75 \mathrm{~V}$ decreases compared to that of the initial sweep, as shown in Fig. 1. The reproducibility of the subsequent $\mathrm{CV}$ s was excellent at sweep rates between 0.2 and $0.005 \mathrm{~V} \mathrm{~s}^{-1}$, indicating that the effect of interference on any electrode reaction such as adsorption can be disregarded.

The proposed mechanism of the oxidation of benzyl alcohols is as follows: ${ }^{5,6}$

$$
\begin{aligned}
& \mathrm{A} \rightleftharpoons \mathrm{A}^{+}+\mathrm{e}^{-} \quad E_{1}^{\mathrm{o}^{\prime}} \\
& \mathrm{A} \cdot+\underset{k_{2}}{\longrightarrow} \mathrm{A} \cdot+\mathrm{H}^{+} \\
& \mathrm{A} \cdot \stackrel{\mathrm{A}^{+}+\mathrm{e}^{-}}{E_{3}^{\mathrm{o}^{\prime}}} \\
& \mathrm{A}^{+} \underset{k_{4}}{\longrightarrow} \mathrm{B}+\mathrm{H}^{+} \\
& \mathrm{A} \cdot^{+}+\mathrm{A} \cdot \underset{k_{5}}{\longrightarrow} \mathrm{A}+\mathrm{A}^{+}
\end{aligned}
$$

The electrooxidation of phenolic aromatic compounds is not initiated by the electron transfer from the phenoxy group, but

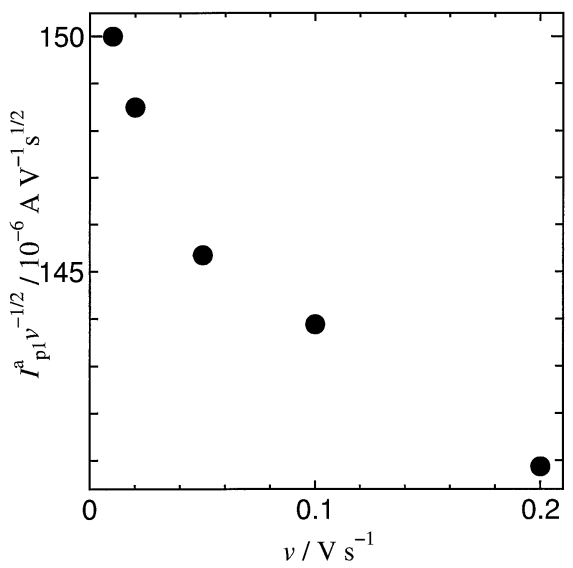

Fig. 2 Variation in normalized peak current for the oxidation of 2 $\mathrm{mM}$ vanillyl alcohol at a Pt electrode with sweep rate.

this transfer comes from the aromatic ring in the case of a compound with strong electron-donating substituents (methoxy moiety in this case) in strongly acidic media $(\mathrm{pH} \leq 2) \cdot{ }^{14}$ Therefore, the first step of the VNA oxidation can be regarded as the formation of the corresponding radical cation, VNA.+ similar to the VA case, ${ }^{5,6}$ though VNA has a phenolic hydroxy group. The electrochemical oxidation of phenolic benzyl alcohol with another substituent group in $o$-position has been reported to form quinone methide compounds by 2-electron process. ${ }^{15}$ The product is the same compound as that obtained in the oxidation by flavo-enzyme, vanillyl alcohol oxidase. ${ }^{16}$ Consequently, the electrooxidation of VNA can be regarded as a 2-electron process and thus mimics enzymatic oxidation. The disappearance of the reduction peak which should be coupled with the oxidation peak at $E_{\mathrm{p}_{1}}^{\mathrm{a}}$ implies that the following chemical reaction, which consumes $\mathrm{VNA}^{+}{ }^{+}$, is too rapid to be observed under the present time scale condition. In aqueous acidic solutions, benzyl alcohol radical cations have been reported to behave as carbon acids coming from $\alpha$-carbon deprotonation, and to form the carbon-centered neutral radicals. ${ }^{17}$ Consequently, the reduction current peak at $E_{\mathrm{p} 2}^{\mathrm{c}}$ are attributable to the reduction of the oxidized product of VNA', which corresponds to species $\mathrm{A}^{+}$in Eq. (3). The existence of the oxidation pre-peak coupled with the reduction peak at $E_{\mathrm{p}_{2}}^{\mathrm{c}}$ at the subsequent potential sweep clearly shows that the electrode process can be interpreted as an ECE (or an ECEC) mechanism in which the redox potentials of the two electroactive species satisfy the relation of $E_{3}^{\mathrm{o}^{\prime}} \ll E_{1}^{\mathrm{o}^{\prime}}$. The cyclic voltammetric shape of this type was theoretically predicted in the early digital simulation study by Feldberg. ${ }^{18,19}$ The plot of the oxidation peak currents at $E_{\mathrm{p}_{1}}^{\mathrm{a}}$ for the initial sweep normalized by the square root of the sweep rate $(v) v s . v$ in Fig. 2 has a negative slope. If the electrode reaction would contain simple electron transfer, the plot for $I_{\mathrm{p}_{1}}^{\mathrm{a}} v^{-1 / 2}$ against $v$ should almost constant. This result guarantees that the deprotonated form of VNA. (VNA.) has a less positive oxidation potential than that of VNA. The summation of the oxidation peak currents at $E_{\mathrm{p} 1}^{\mathrm{a}}$ and $E_{\mathrm{p} 2}^{\mathrm{a}}$ for the subsequent potential sweep is almost equal to the peak current at $E_{\mathrm{p}_{1}}^{\mathrm{a}}$ for the initial sweep, suggesting that the second oxidation step in Eq. (3) might not be influenced by any disturbance such as adsorption nor by the following chemical reactions. In contrast to the first deprotonation process in Eq. (2), the deprotonation process in Eq. (4) is considered to be negligibly sluggish in a strong acidic aqueous solution. Therefore, we could treat the reaction as an ECE mechanism but 
(a)

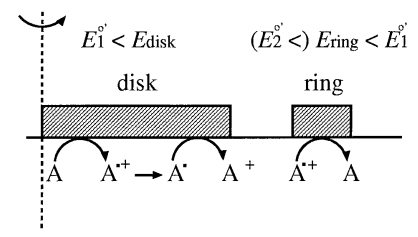

(b)

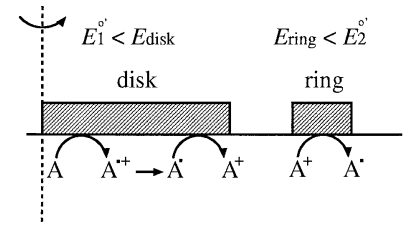

Fig. 3 Schematic reaction pathway for the collection at the ring electrodes of different potentials.

not as an ECEC mechanism. It was reported that electrochemical formation of radical cations of methoxybenzyl alcohols is initiated by a reversible electron transfer in basic aprotic solvents. ${ }^{20,21}$ The peak separation between $E_{\mathrm{p} 2}^{\mathrm{a}}$ and $E_{\mathrm{p} 2}^{\mathrm{c}}$ indicates that the second heterogeneous electron transfer is less facile than the first oxidation step.

To resolve the heterogeneous electron transfer steps, which put a first chemical reaction between them, we carried out the steady-state RRDE voltammetries at different ring potentials, which enabled us to distinguish two electroactive species, one generated by the electrode reaction at the disk electrode and the other by the following chemical reaction. The scheme for the collection at the ring electrodes is indicated in Fig. 3. For the analysis of the simple EC reaction, process (a) has been used exclusively. ${ }^{22,23}$ In the present case, where we treat the reaction as the potential-reversed ECE mechanism, process (b) was attempted. Figure 4 shows the typical steady-state current-potential curves for the oxidation of $2 \mathrm{mM} \mathrm{VNA}$ at a $\mathrm{Pt}$ RRDE. The disk potential $\left(E_{\mathrm{d}}\right)$ was swept at $0.01 \mathrm{~V} \mathrm{~s}^{-1}$ from 0.5 $\mathrm{V}$. Curve $\mathrm{b}$ is a ring current $\left(I_{\mathrm{r} 2}\right) v s$. $E_{\mathrm{d}}$ trace. The ring potential in curve b was maintained at a constant value of $0.2 \mathrm{~V}\left(E_{\mathrm{r} 2}\right)$, which was sufficiently negative to $E_{\mathrm{p}_{2}}^{\mathrm{c}}$ in Fig. 1 . An increase in the ring current results from the reduction of the final product being formed at the disk electrode (species $\mathrm{A}^{+}$in Eq. (4)). The limiting current of curve a at the disk electrode $\left(I_{\lim \mathrm{d}}\right)$ constitutes the summation of the oxidation processes in Eqs. (1) and (3). As indicated by the $\mathrm{CV}$ in Fig. 1, since the first-order-following chemical reaction which consumes $\mathrm{VNA}^{+}{ }^{+}$is significantly facile, the limiting current in the process in Eq. (1), $I_{\lim (1)}$, can be expressed by the following equation:

$$
I_{\lim (1)}=I_{\operatorname{lim~d}}-I_{\operatorname{lim~r2}} / N
$$

where $N$ is the collection efficiency of the RRDE and $I_{\lim \mathrm{r} 2}$ is the limiting current at the ring electrode whose potential was constant at $0.2 \mathrm{~V}$. In this case, we make the assumption that the process in Eq. (3) would not be perturbed by any chemical reactions, that is, the reaction mechanism could be treated not as an ECEC mechanism but as an ECE mechanism. The typical Levich plots of the limiting currents, $I_{\operatorname{lim~d}}, I_{\lim (1)}$ and $I_{\lim \mathrm{r} 2} v s$. (rotation rate $\omega)^{1 / 2}$ are shown in Fig. 5. The dependence of values of $I_{\lim (1)}$ on $\omega^{1 / 2}$ indicates that the process obeys the Levich equation: ${ }^{24}$

$$
I_{\lim (1)}=0.62 n F A D^{2 / 3} v^{-1 / 6} c \omega^{1 / 2}
$$

where $c$ is the bulk concentration of VNA, $D$ is the diffusion coefficient of VNA, $v$ is the kinematic viscosity of the solution (the value of $0.01 \mathrm{~cm}^{2} \mathrm{~s}^{-1}$ for water was assumed and used here), ${ }^{25,26} n$ is the number of electrons transferred (the value of 1.0 was used), $A$ is the electrode area and $F$ is Faraday's constant. The deviation from the straight line in the $I_{\lim r 2}$ case is

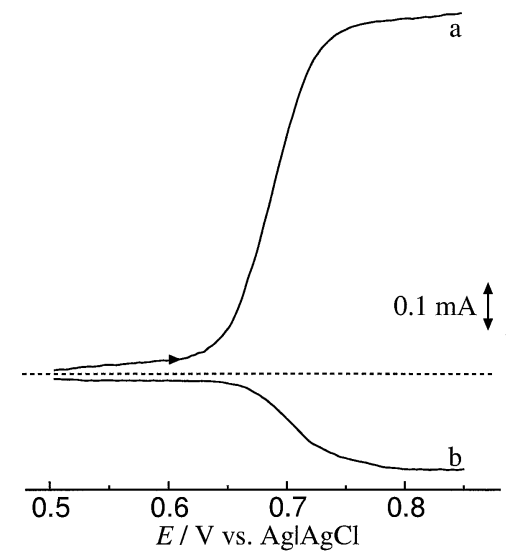

Fig. 4 Steady-state current-potential curves for the oxidation of 2 $\mathrm{mM}$ vanillyl alcohol at rotating ring-disk Pt electrode. Rotation rate: $200 \mathrm{rpm}$. Ring electrode potential: (b) $0.2 \mathrm{~V} v s$. $\mathrm{Ag} \mid \mathrm{AgCl}$. The disk potential was swept at $0.01 \mathrm{~V} \mathrm{~s}^{-1}$

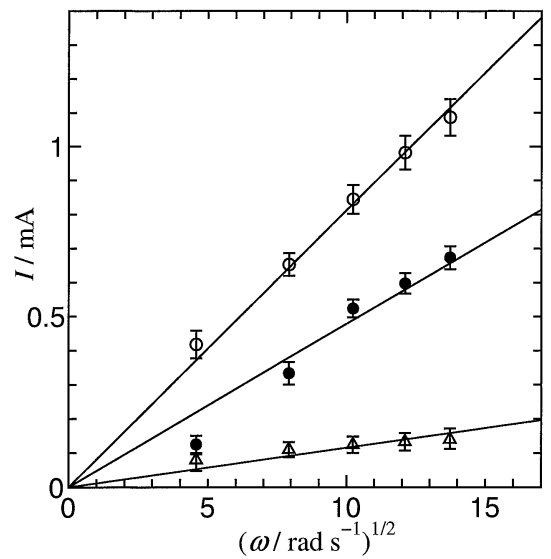

Fig. 5 Levich plots of the limiting current, $I_{\text {lim }} v s$. (rotation rate, $w)^{1 / 2}$ for the steady-state current-potential curves for the oxidation of vanillyl alcohol at the rotating ring-disk electrode. (O) $I_{\mathrm{d}},(\bullet) I_{\lim (1)}=$ $I_{\operatorname{lim~d}}-I_{\operatorname{lim~r} 2} / N,(\triangle) I_{\operatorname{lim~r2} .}$

probably due to the gap between the ring and disk electrodes which is slightly too wide for the detection of short-lived radical species $(N=0.347)$. From the slope of the Levich plot in Fig. 5, the value of $D_{\mathrm{VNA}}$ were determined to be $(7.2 \pm 0.5) \times 10^{-6} \mathrm{~cm}^{2}$ $\mathrm{s}^{-1}$.

In conclusion, the electrochemical oxidation of vanillyl alcohol in a strong acidic aqueous solution proceeds as an ECE mechanism in which sluggish electron transfer after deprotonation of a radical cation intermediate occurs at a less positive potential than that of the initial oxidation. If the material is to be an efficient 1-electron redox mediator from enzymes such as lignin peroxidase and vanillyl alcohol oxidase to the substrate lignin polymer, a long-lifetime of radical cation intermediates is essential besides having high redox potential. Many aromatic methoxy compounds might satisfy the above conditions and we could add the sluggish electron transfer rate of neutral radical species for aromatic methoxy compounds.

\section{References}

1. M. Tien and T. K. Kirk, Science, 1983, 221, 661 . 
2. P. J. Kersten, M. Tien, B. Kalyanaraman, and T. K. Kirk, J. Biol. Chem., 1985, 260, 2609.

3. P. J. Harvey, H. E. Schoemaker, and J. M. Palmer, FEBS Lett., 1986, 195, 242.

4. D. C. Goodwin, S. D. Aust, and T. A. Grover, Biochemistry, 1995, 34, 5060.

5. D. T. Sawyer, A. Sobkowiak, and J. L. Roberts, Jr., "Electrochemistry for Chemists", 2nd ed., 1995, John Wiley \& Sons, New York, 461.

6. S. Kishioka and A. Yamada, Chem. Lett., 2004, 33, 408.

7. A. Khindaria, I. Yamazaki, and S. D. Aust, Biochemistry, 1996, 35, 6418.

8. M. Bietti, E. Baciocchi, and S. Steenken, J. Phys. Chem. A, 1998, 102, 7337.

9. S. Kishioka, M. Umeda, and A. Yamada, 203rd Meeting of The Electrochemical Society, Paris, France, 2003, AC1, Abstract 2474.

10. M. C. Boyce, P. R. Haddad, and T. Sostaric, Anal. Chim. Acta, 2003, 485, 179.

11. P. Parpot, A. P. Bettencourt, A. M. Carvalho, and E. M. Belgsir, J. Appl. Electrochem., 2000, 30, 727.

12. K. B. Prater and A. J. Bard, J. Electrochem. Soc., 1970, 117, 335.

13. K. B. Prater and A. J. Bard, J. Electrochem. Soc., 1970, $117,1517$.

14. J. Stradins and B. Hasanli, J. Electroanal. Chem., 1993,
353, 57

15. V. V. Yanilkin, V. A. Medvedev, V. A. Babkin, S. Z Ivanova, and S. A. Medvede, Izv. Akad. Nauk. SSSR, Ser. Khim., 1985, 132.

16. R. H. H. van den Heuvel, M. W. Fraaije, and W. J. H. van Berkel, Methods in Enzymology, 2002, 353, 177.

17. E. Baciocchi, M. F. Gerini, O. Lanzalunga, and S. Mancinelli, Tetrahedron, 2002, 58, 8087.

18. S. W. Feldberg, J. Phys. Chem., 1971, 75, 2377.

19. A. J. Bard and L. R. Faulkner, "Electrochemical Methods, Fundamentals and Applications", 2nd ed., 1994, Chap. 12, John Wiley \& Sons, New York.

20. O. R. Brown, S. Chandra, and J. A. Harrison, J. Electroanal. Chem., 1972, 34(2), 505.

21. O. R. Brown, S. Chandra, and J. A. Harrison, J. Electroanal. Chem., 1972, 38(1), 185.

22. W. J. Albery and S. Bruckenstein, Trans. Faraday Soc., 1966, 62, 1946.

23. W. J. Albery and M. L. Hitchman, "Ring-disc Electrodes", 1971, Oxford University Press, Glasgow.

24. V. G. Levich, "Physicochemical Hydrodynamics", 1962, Prentice-Hall, Englewood Cliffs, NJ, 345.

25. R. R. Durand and F. C. Anson, J. Electroanal. Chem., 1982, 134, 273.

26. M. Tsushima, K. Tokuda, and T. Ohsaka, Anal. Chem., 1994, 66, 4551. 